\title{
Epicenter Localization Using RDOA based RLS Algorithm
}

\author{
Younghun Pyo, Kyunghyun Lee, Kwanho You* \\ Department of Electrical and Computer Engineering, Sungkyunkwan University, South Korea
}

Copyright $(2017$ by authors, all rights reserved. Authors agree that this article remains permanently open access under the terms of the Creative Commons Attribution License 4.0 International License

\begin{abstract}
In this paper, we propose an epicenter measurement using a laser interferometer. The laser interferometer system is an equipment with its extraordinary accurate capability for displacement measurement. The seismic signal is measured in the observation as $\mathrm{P}$-wave and $\mathrm{S}$-wave form. The seismic signal is indicated as in a form of intensity signal which is represented as a trigonometrical function using the heterodyne laser interferomter. The epicenter can be derived by P-S time which can be determined by intensity signal. With a range difference of arrival (RDOA) method, we can estimate the epicenter location from the distance data between the epicenter and the observatory. We suggest a recursive least square (RLS) algorithm to obtain a solution that minimizes estimation error value. Through some simulations, we prove the performance of the proposed method.
\end{abstract}

Keywords Heterodyne Laser Interferometer, P-S Time, Range Difference of Arrival, Recursive Least Square

\section{Introduction}

Globally, the damage from the earthquake has been increasing around the world. Analyses of earthquakes have been extended to diverse parts such as distance of epicenter, magnitude, frequency measurement and intensity analysis. The main purpose of the studies about the earthquake is to reduce the damage caused by an earthquake. The more studies deeply are performed, the more accurate measurement equipment is required. To enhance precise seismic wave measurement, we use a laser interferometer. The seismic wave is mainly measured by a velocimeter or an accelerometer in the earthquake analysis. If the measured seismic signal is more accurate, it is possible to reduce the damage of the earthquake. There have been many studies to detect and analyze the earthquakes.

Amin [1] proposed a method for early detection of earthquakes using the notion of quantum computing. This method can measure the slightest change in the gravity field of the particular place using the notion of quantum computing. Rastin [2] suggested a method to measure simplified earthquake event waveforms (SEEW) of local earthquakes from the seismic signals. Prasad [3] proposed a method to measure earthquake using primary waves and secondary waves. The parameters of seismic signal are analyzed by using fast fourier transform. The magnitude which types the base of analysis is used for the detection of earthquake.

In this paper, we propose an accuracy epicenter measurement solution with a heterodyne laser interferometer that determines epicenter location. The heterodyne laser interferometer measures a seismic wave more accurately than seismometers such as accelerometers and velocimeters. Using the heterodyne laser interferometer, the seismic signals are indicated as in a form of intensity signal which is represented as a trigonometrical function. The P-S time is determined and the epicenter can be derived by intensity signal. RDOA method is used to measure the epicenter. Using the difference of the distances which are measured from several separate observatories, we can measure the epicenter location more accurately. To obtain a solution that minimizes the estimation error value, we propose the RLS algorithm.

This paper is organized as follows. In section 2, a measurement of seismic signal detection with the heterodyne laser interferometer is introduced. In section 3, we measure the epicenter location with the RDOA method and explain an error compensation with the RLS algorithm. Section 4 shows simulation results of the proposed method. Finally, conclusions are presented in section 5.

\section{P-S Time Measurement Using Heterodyne Laser Interferometer}

The laser head emits two beams with different frequencies which are orthogonal and polarized to each other in heterodyne laser interferometer. Figure 1 presents a heterodyne laser interferometer system. 


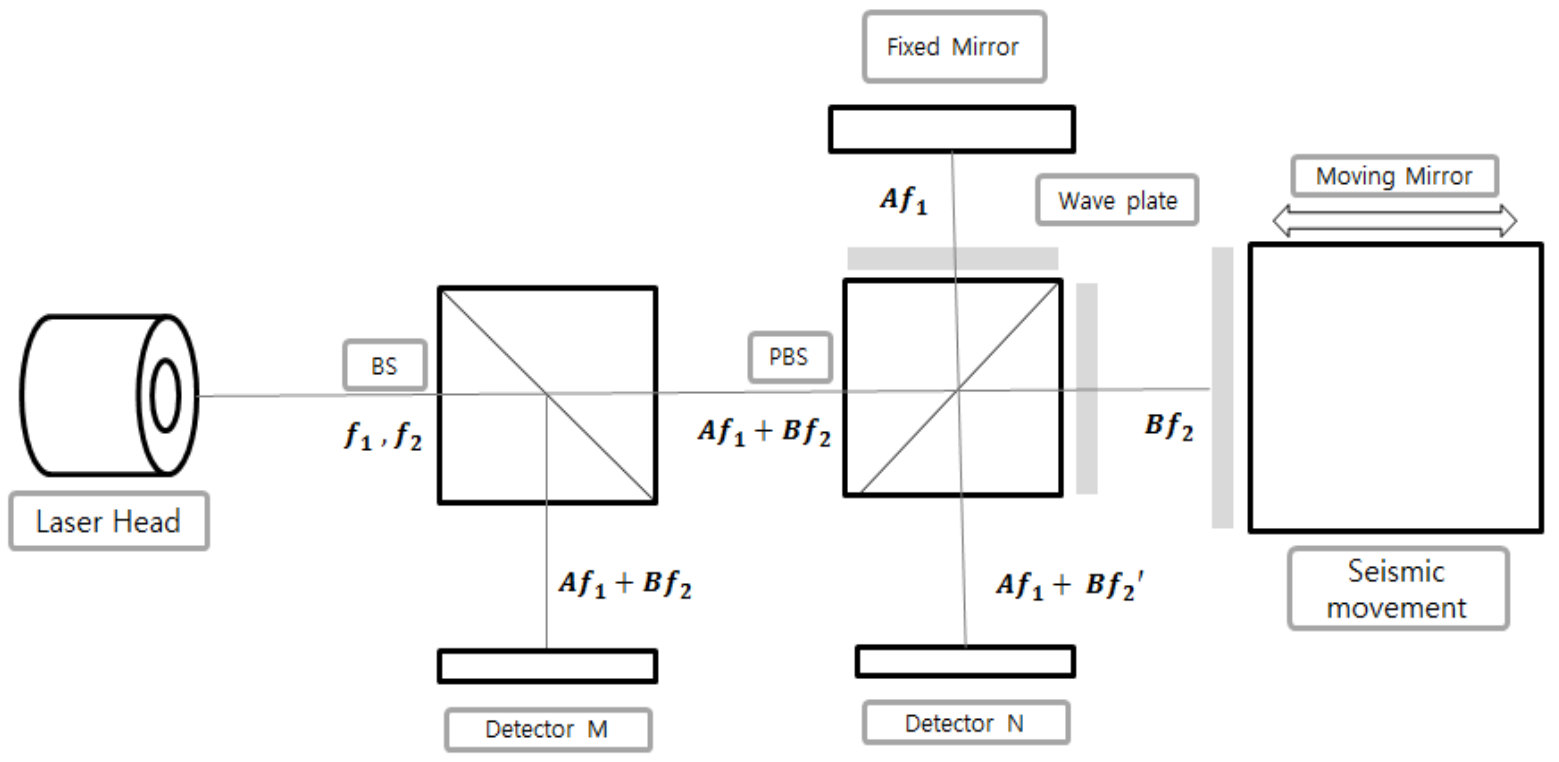

Figure 1. Heterodyne laser interferometer based seismic detection system

The laser source is divided into two signals which are reference and measurement signals while they pass through a beam splitter (BS). The reference signal is collected by detector $\mathrm{M}$. The photo detector $\mathrm{M}$ measures the output signal $I_{r}$

$$
\begin{aligned}
I_{r} & \propto\left(P_{M 1}+P_{M 2}\right)\left(P_{M 1}+P_{M 2}\right)^{*} \\
& =\frac{1}{2}\left(A^{2}+B^{2}\right)+A B \cos \left[2 \pi \Delta f t+\left(\Phi_{B}-\Phi_{A}\right)\right]
\end{aligned}
$$

where $A$ and $B$ are the amplitude of electric field, and $\Phi_{A}, \Phi_{B}$ are the initial phase values. The measurement signal is divided by a polarizing beam splitter (PBS). One is reflected by a moving mirror, and the other is reflected by a fixed mirror. The reunified signal is collected by detector $\mathrm{N}$ through the polarizing beam splitter. The photo detector $\mathrm{N}$ measures the output signal $I_{m}$

$$
\begin{aligned}
I_{m} & \propto\left(P_{N 1}+P_{N 2}\right)\left(P_{N 1}+P_{N 2}\right)^{*} \\
& =\frac{1}{2}\left(A^{2}+B^{2}\right)+A B \cos \left[2 \pi \Delta f t+\left(\Phi_{B}-\Phi_{A}\right)+\Delta \Phi\right]
\end{aligned}
$$

where $\Delta \Phi$ is the phase difference which is occurred by Doppler effect. The DC components of reference and measurement signal are eliminated by a high-pass filter. A lock-in amplifier is used in order to obtain the phase value. Then the final calculated signals are represented in sine and cosine waveforms.

$$
\begin{aligned}
& I_{x} \propto \frac{A B}{2} \cos \Delta \Phi \\
& I_{y} \propto \frac{A B}{2} \sin \Delta \Phi
\end{aligned}
$$

The phase difference $\Delta \Phi$ can be obtained by a trigonometrical function. Using the phase difference, the distance change $\Delta L$ between fixed mirror and moving mirror is acquired.

$$
\begin{aligned}
& \Delta \Phi=\tan ^{-1}\left(\frac{I_{y}}{I_{x}}\right) \\
& \Delta L=\frac{\Delta \Phi \lambda}{4 \pi n}
\end{aligned}
$$

where $n$ is an air refractive index and $\lambda$ represents the mean of wavelengths. The seismic signal is measured in the observation as $\mathrm{P}$-wave and $\mathrm{S}$-wave form. According to the differences of amplitude and velocity between P-wave and $\mathrm{S}$-wave, the arrival time of $\mathrm{P}$-wave and $\mathrm{S}$-wave can be determined. Difference of the arrival time of the first P-wave and S-wave is called as P-S time. The distance from an observatory to the epicenter can be measured by the following equation [4].

$$
D_{\varepsilon}=\frac{v_{p} v_{s} t_{p s}}{v_{p}-v_{s}}
$$

where $v_{p}$ and $v_{s}$ denotes the velocity of P-wave and S-wave, respectively. $t_{p s}$ represents the P-S time.

\section{Epicenter Determination and Error Compensation}

RDOA method is one of the most commonly used method in the location decision. Using the RDOA method, we can measure the distance from the epicenter to the observatory. The RDOA equation can be expressed as follows.

$$
d_{i}=\left\|m-b_{i}\right\|, i=1,2, \ldots, M
$$


where $m=[x, y]^{T}$ and $b_{i}=\left[x_{i}, y_{i}\right]^{T}$ are the unknown location of epicenter and the known location of $i$-th observatory, respectively. In real condition, the measured RDOA data contains measurement error due to environmental noise. Under the assumption that the RDOA measurements do not contain environmental noise, we can obtain the following set of linear equations.

$$
\begin{aligned}
& \left(x-x_{1}\right)\left(x_{i}-x_{1}\right)+\left(y-y_{1}\right)\left(y_{i}-y_{1}\right)+d_{i 1} d_{1} \\
& =\frac{1}{2}\left[\left(x_{i}-x_{1}\right)^{2}+\left(y_{i}-y_{1}\right)^{2}-d_{i 1}^{2}\right]
\end{aligned}
$$

The equation (7) which demonstrates the relation between RDOA and the location of epicenter can be rewritten as a following matrix form,

$$
A u=b
$$

with

$$
\begin{gathered}
A=\left[\begin{array}{ccc}
x_{2}-x_{1} & y_{2}-y_{1} & d_{21} \\
\vdots & \vdots & \vdots \\
x_{M}-x_{1} & y_{M}-y_{1} & d_{M 1}
\end{array}\right], \\
b=\frac{1}{2}\left[\begin{array}{c}
\left(x_{2}-x_{1}\right)^{2}+\left(y_{2}-y_{1}\right)^{2}-d_{21}^{2} \\
\vdots \\
\left(x_{M}-x_{1}\right)^{2}+\left(y_{M}-y_{1}\right)^{2}-d_{M 1}^{2}
\end{array}\right]
\end{gathered}
$$

where the parameter vector $u=\left[\begin{array}{lll}x-x_{1} & y-y_{1} & d_{1}\end{array}\right]^{T}$ is the solution of RDOA. Also we propose the RLS algorithm to compensate a measurement error [5]. The object function for minimizing error between the epicenter estimation and real one can be obtained as a following equation.

$$
f(\hat{u})=(A \hat{u}-b)^{T}(A \hat{u}-b)
$$

In equation (10), $\hat{u}$ denotes the estimated value of variable $u$. We need to obtain the least square (LS) algorithm of equation (10) in order to find the RLS algorithm solution. The LS solution that minimizes the sum of error resulted from each single equation can be expressed as follows,

$$
\hat{u}=\left(A^{T} A\right)^{-1} A^{T} b
$$

We need to represent a system equation in a different form in order to apply RLS algorithm. Equation (8) can be rewritten as,

$$
\left(\begin{array}{l}
\bar{A}_{k} \\
A_{k+1}
\end{array}\right) u_{k+1}=\left(\begin{array}{l}
\bar{b}_{k} \\
b_{k+1}
\end{array}\right)
$$

In equation (12), $\bar{b}_{k}$ and $\bar{A}_{k}$ express the values which contain $k^{\prime} s$ past measurement data. $b_{k+1}$ and $A_{k+1}$ mean $(k+1)$-th measured seismic data. $\hat{u}_{k+1}$ can be obtained through the LS algorithm as follows,

$$
\hat{u}_{k+1}=\left(\bar{A}_{k+1}^{T} \bar{A}_{k+1}\right)^{-1} \bar{A}_{k+1}^{T} \bar{b}_{k+1}
$$

With the definition of $G_{k+1}=\left(\bar{A}_{k+1}^{T} \bar{A}_{k+1}\right)^{-1}$ for a simple notation, $G_{k+1}^{-1}$ can be represented as following,

$$
G_{k+1}^{-1}=G_{k}^{-1}+A_{k+1}^{T} A_{k+1}
$$

Using the equation (14), the equation (13) is rewritten as follows,

$$
\begin{aligned}
& \hat{u}_{k+1}=G_{k+1}\left[\bar{A}_{k}^{T} \bar{b}_{k}+A_{k+1}^{T} b_{k+1}\right] \\
& =G_{k+1}\left[G_{k}^{-1} G_{k} \bar{A}_{k}^{T} \bar{b}_{k}+A_{k+1}^{T} b_{k+1}\right]
\end{aligned}
$$

The equation (15) can be rewritten as follows,

$$
\begin{aligned}
\hat{u}_{k+1} & =G_{k+1}\left[G_{k}^{-1} \hat{u}_{k}+A_{k+1}^{T} b_{k+1}\right] \\
& =G_{k+1}\left[\left(G_{k+1}^{-1}-A_{k+1}^{T} A_{k+1}\right) \hat{u}_{k}+A_{k+1}^{T} b_{k+1}\right] \\
& =\hat{u}_{k}-G_{k+1} A_{k+1}^{T} A_{k+1} \hat{u}_{k}+G_{k+1} A_{k+1}^{T} b_{k+1} \\
& =\hat{u}_{k}+G_{k+1} A_{k+1}^{T}\left(b_{k+1}-A_{k+1} \hat{u}_{k}\right)
\end{aligned}
$$

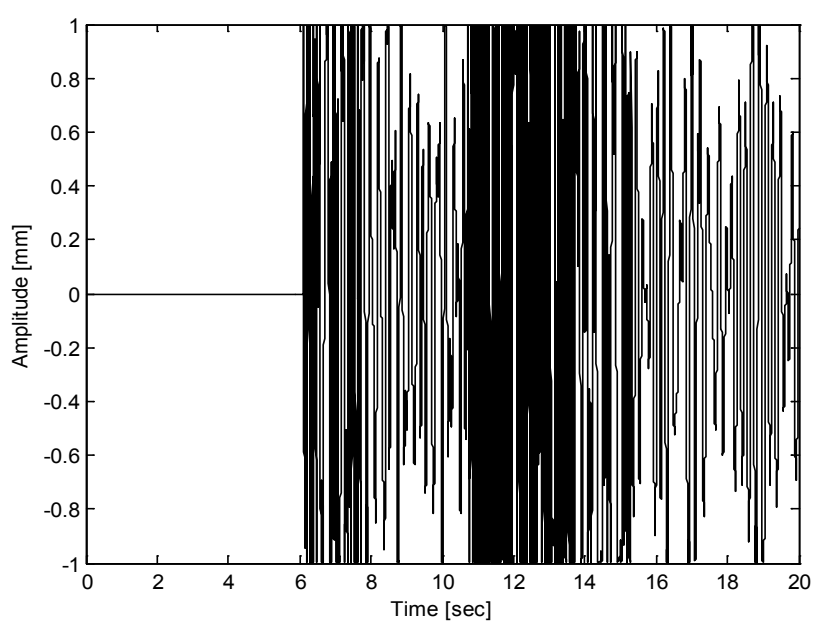

Figure 2. Intensity signal $I_{y}$ for spectrum analysis

Optimization solution of the objective function is derived. Using the equation (16), we prove the effectiveness of RLS algorithm by showing the accurate epicenter location. RLS algorithm has an advantage to find the new epicenter location more accurately and rapidly than LS algorithm.

\section{Simulation Results}

In this section, we demonstrate the measurement of a seismic signal by using a laser interferometer and measure the P-S time using the intensity signal. Through some simulations, we also measure the distance from the epicenter to the observatory and determine epicenter location. 
Wavelength $\lambda$ is set as $632.991 \mathrm{~nm}$ and the air refractive index $n$ is 1.00000002665 . In our simulation, we can measure the seismic wave movement using the laser interferometer. Figure 2 shows the intensity signal $I_{y}$ for spectrum analysis. The high density points of intensity signal can be represented as the arrival time of $\mathrm{P}$-wave and $\mathrm{S}$-wave around 6 and 11 seconds, respectively.

Figure 3 shows that the root mean square error (RMSE) of epicenter location is decreased with the increased number of observatories. The RMSE of estimated position is demonstrated for the increased number of observatory. When additional RDOA data is measured from new observatory, the accuracy of epicenter location is improved and can be quickly estimated by using previous calculated epicenter location and additional RDOA data through our proposed RLS algorithm.

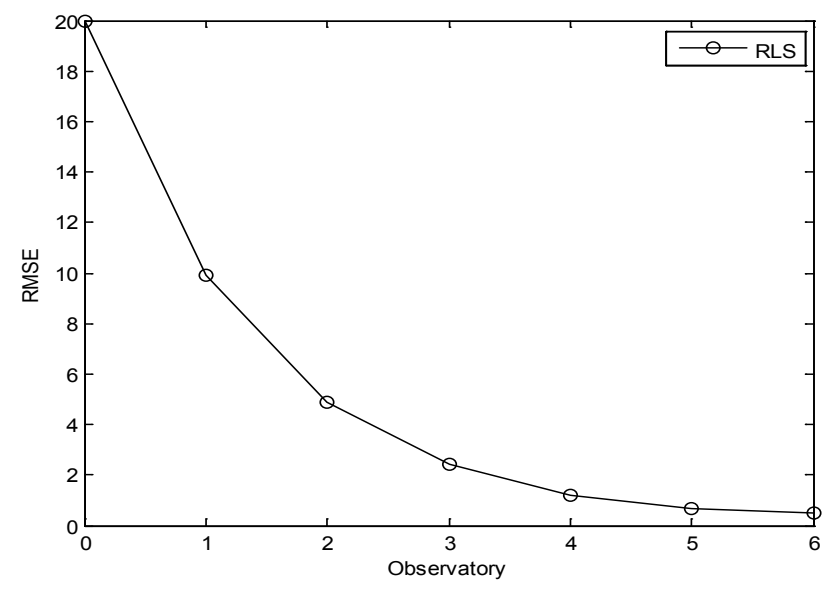

Figure 3. RMSE change for observatory

\section{Conclusions}

In this paper, we proposed an RDOA method to measure epicenter location and an RLS algorithm to increase the epicenter location accuracy. We measured P-S time from intensity signal using the heterodyne laser interferometer. We measured the location of epicenter using the RDOA method which is the location estimation method using difference of distance from epicenter to each observatory. Finally, we suggested the RLS based optimization scheme for minimizing the estimation error of epicenter location. The effectiveness of our proposed algorithm was confirmed through some simulations.

\section{Acknowledgements}

This work was supported by the National Research Foundation of Korea (NRF) grant funded by the Korea government (MSIP) (NRF-2016R1A2B4011369).

\section{REFERENCES}

[1] Amin, A. A., \& Hasan, H. Early detection of earthquake using satellite based quantum computing, International Conference on Computer Science and Network Technology, 1079-1082, 2015.

[2] Rastin, S. J., \& Unsworth, C. P. Iterative coupling of standardized earthquake detection \& wavelet thresholding to determine simplified earthquake event waveforms, Information Sciences Signal Processing and their Applications, 173-176, 2010.

[3] Prasad, N. \& Murthy, S. Analysis of magnitude for earthquake detection using primary waves and secondary waves, Human Computer Interactions, 1-6, 2013.

[4] Gustafasson, F. \& Gunnarsson, F. Positioning using time difference of arrival measurements, IEEE Int. Conf. Acoust. Speech. Signal Process., 553-556, 2003

[5] Olyaee, S. \& Abadi, M. Use of adaptive RLS, LMS, and NLMS algorithms for nonlinearity modeling in a modified laser interferometer, Frontiers of Optoelectronics. China, 3(3), 264-269, 2010. 